\title{
PRETERM TWINS AND TRIPLETS ARE AT INCREASED RISK FOR THE DEVELOPMENT OF PERIVENTRICULAR LEUKOMALACIA
}

\author{
B. Resch, E. Resch, T. Freidl, A. Brandner, U. Maurer, F. Reiterer, W. Müller
}

Medical University of Graz, Graz, Austria

Objective: To determine the risk for the development of periventricular leukomalacia (PVL) of twins and triplets compared to singletons.

Methods: Retrospective single-centre study including all infants $\leq 35$ weeks of gestational age with cystic PVL documented by ultrasound scans between 1988 and 2008.

Results: Of 4926 singleton births, 579 twins, 37 triplets, and one quintuplet (excluded from further analysis) 117 singletons and 39 multiples were diagnosed as having cystic PVL. Perinatal data did not differ between groups as did not ultrasonographic findings and neurodevelopmental outcome (cerebral palsy rate 81 vs. 77\%). The relative risk (RR) for twin pregnancy resulting in preterm birth and having at least one infant with PVL was 2.181 (CI 95\% $1.474-3.228, \mathrm{p}<.0001$ ), and for triplet pregnancy 6.793 (CI 95\% $2.470-13.108$, $\mathrm{p}<.0001)$. The RR increased by $2.608(1.075-6.329, \mathrm{p}=.017)$ from twin to triplet pregnancy. Multiples had a higher rate of caesarean section $(73 \mathrm{vs.} 44 \%, \mathrm{p}<.001)$, less often preterm premature rupture of the membranes ( 33 vs. $58 \%, p<.006)$ and clinical chorioamnionitis $(28$ vs. $46 \%, p=.039)$, and fewer diagnoses of fetal distress (18 vs. 34\%, $<<.001)$, hyperbilirubinemia (23 vs. 53\%, $\mathrm{p}<.001)$ and intraventricular hemorrhage grade 2 ( 0 vs. $9 \%, p=.024)$.

Conclusions: Our findings demonstrate an increased risk for the development of PVL in twin and triplet pregnancy. No overt risk factors were found in multiples compared to singletons. 